\title{
MEDIASTINAL TERATOMATA
}

\author{
BY \\ B. T. Le ROUX \\ From the Department of Surgery, University of Edinburgh
}

(RECEIVED FOR PUBLICATION MAY 30, 1960)

Teratology, unencumbered by fanciful hypotheses such as parthenogenesis and the development of the "foetus in foetu," remains an arresting study because of the bizarre structure of many teratomata. A teratoma is a true tumour composed of multiple tissues foreign to the part in which the tumour is found (Willis, 1953). In contradistinction to a quiescent malformation, a teratoma displays progressive, uncoordinated growth. The multiplicity of tissues contained in a teratoma may be demonstrated only by the most detailed examination, but is nevertheless evident in even the simplest dermoid cyst (Schlumberger, 1951), which is no more than a highly differentiated teratoma composed of relatively quiescent tissues of mature adult type. That the tissues comprising a teratoma are foreign to the part in which it is found is evident when the tumour occurs in the mediastinum, from which it can often be shelled merely by the incision of the pleural reflection covering it without the division of any pedicle and without the resection of any related structure in the mediastinum.

Subdivisions into cystic and solid growths, of mono-, bi-, or tri-dermal origin, depending on the representation of "germ" layers in them, are artificial. Division of teratomata into those containing mature tissues and those containing embryonic tissues may be of value, since the latter are usually malignant tumours and the former benign, but the distinction remains arbitrary, because mixtures of adult and embryonic tissues of all degrees of maturity are found.

Histogenetically teratomata are regarded as tumours arising from foci of plastic pleuripotential embryonic tissue which has escaped from the influence of primary organizers during early embryonic development. This escape is in some way related to disturbances emanating from the invaginated organizing tissues of the primitive streak, and median and paramedian parts are therefore affected. Mediastinal teratomata, which Willis maintains are always anterior, are believed more specifically to arise from cells derived from the branchial remnants in connexion with the thymus, which originates from the third branchial pouch and the overlying third branchial cleft. Lesions called teratomata in the posterior mediastinum are either notochordal remnants, or they have developed in association with deformities of the chest which have so distorted the normal relationships that anterior mediastinal structures are displaced posteriorly (Morrison, 1958).

The experience in the Regional Thoracic Unit in Edinburgh of teratomata is shown in the accompanying table. Nineteen patients were submitted to thoracotomy for anterior mediastinal lesions shown by subsequent histological examination to be teratomata. Two patients are believed still to harbour such a tumour. One declined thoracotomy (Case 1): the opacity in this patient has been known to be present for 14 years. The other (Case 2) bears in addition the stigmata of dystrophia myotonica, the manifestations of which have been observed to progress over eight years without change in the anterior mediastinal opacity, and from which he is believed to be in greater danger than from his likely teratoma. There is sound radiographic evidence in these two patients for classifying their lesions as teratomata : a rind of calcium in one, and recognizable teeth within the opacity in the other.

The age of the youngest patient whose teratoma was resected was 15 years and the oldest 65 years. The sexes are nearly equally represented. Twelve tumours were right-sided, but in two of these a small part of the opacity was visible to the left of the mediastinum. Nine tumours were left-sided, and in one of these a small part of the opacity extended to the right of the mediastinum.

In 13 patients the lesion was detected by routine radiography. None of these patients had symptoms. Two patients (Cases 7 and 14) were investigated because of recurring respiratory infections, during which hair and greasy material were coughed up. In the youngest patient (Case 4) a respiratory illness during childhood had prompted chest radiography; an opacity interpreted as a pleural effusion had been found, and sebaceous material had been aspirated from this. 
The remaining five patients had been radiographed because of complaints of pain or dyspnoea or both.

One of the patients with trichoptysis has clubbed fingers. In the remaining 20 the fingers were normal. Anterior dullness to percussion was elicited in eight patients with large tumours. In five of these there was visible an anterior bulging of ribs and costal cartilages overlying the opacity.
In two women a loud systolic murmur was heard in the pulmonary area with the patient in the recumbent position. The murmur became softer when the patients leaned forward, and disappeared with resection of the tumours. No other abnormalities were found on examination.

All the opacities lay in the anterior mediastinum and were clearly visible on postero-anterior and lateral radiographs. In seven patients the radio-

TABLE

CLINICAL SUMMARY OF SERIES

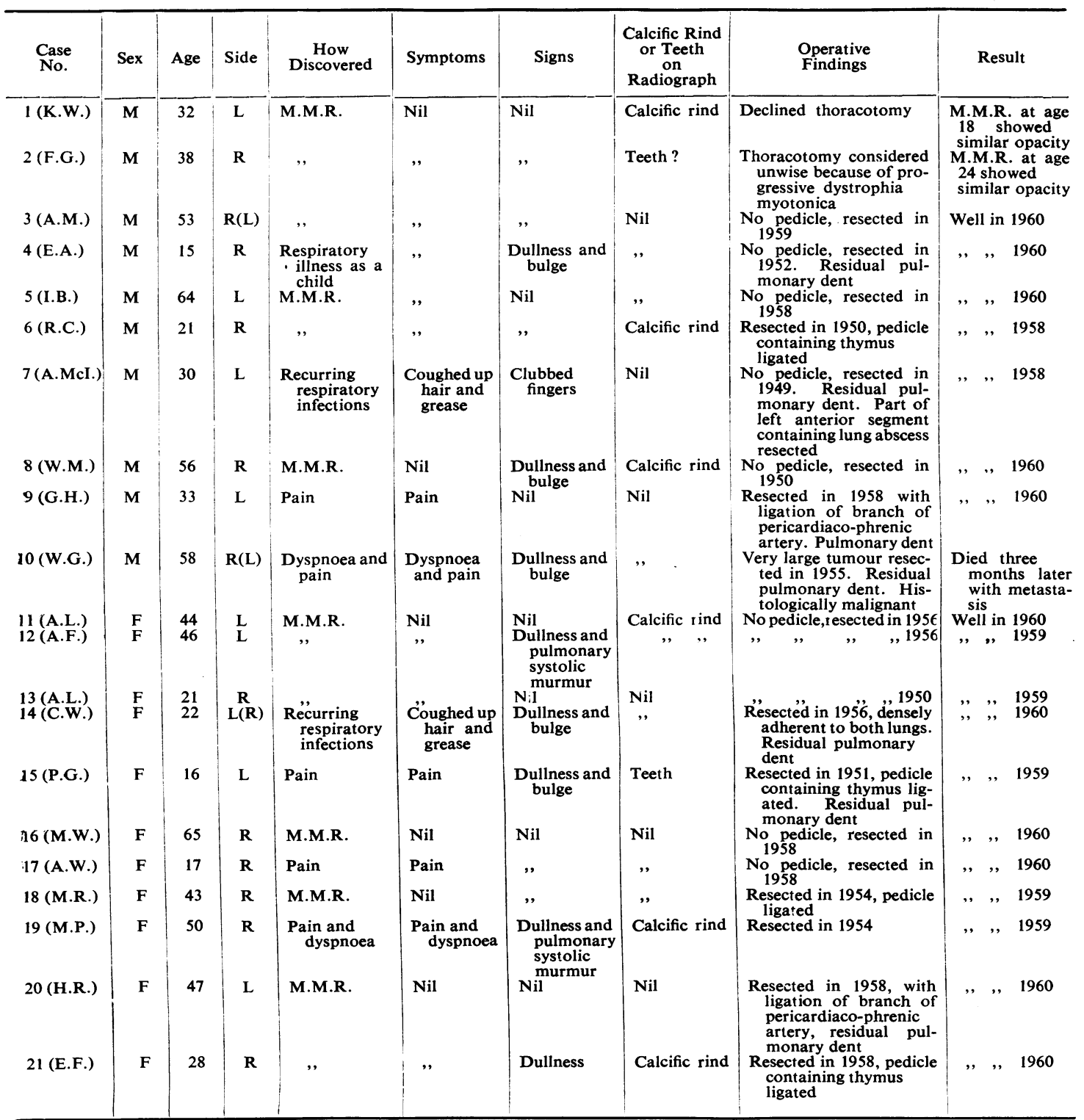




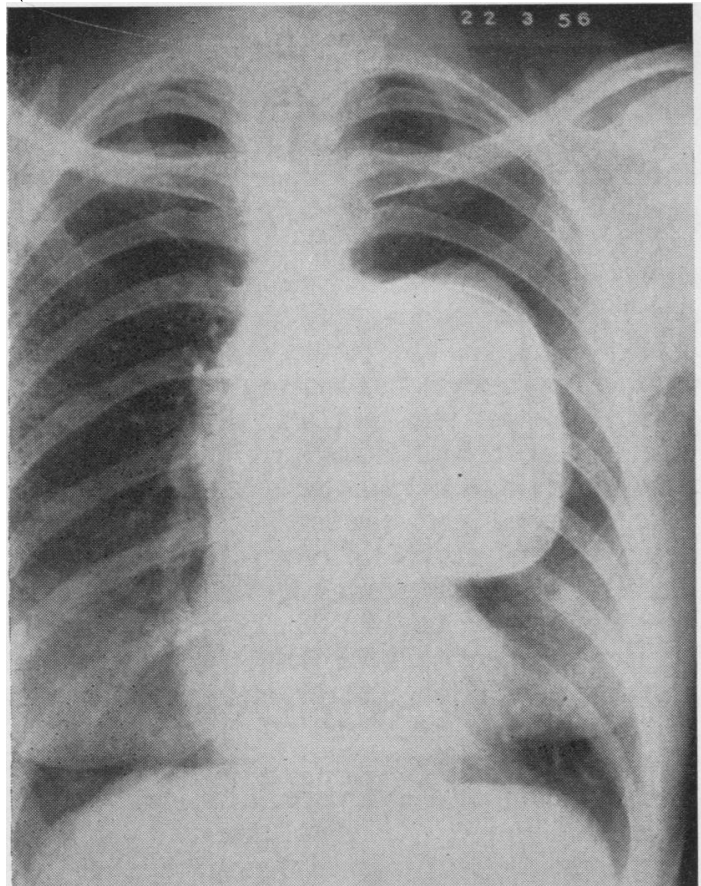

Fig. 1.-Postero-anterior view showing the calcific rind $\iota$ bout a large mediastinal teratoma.

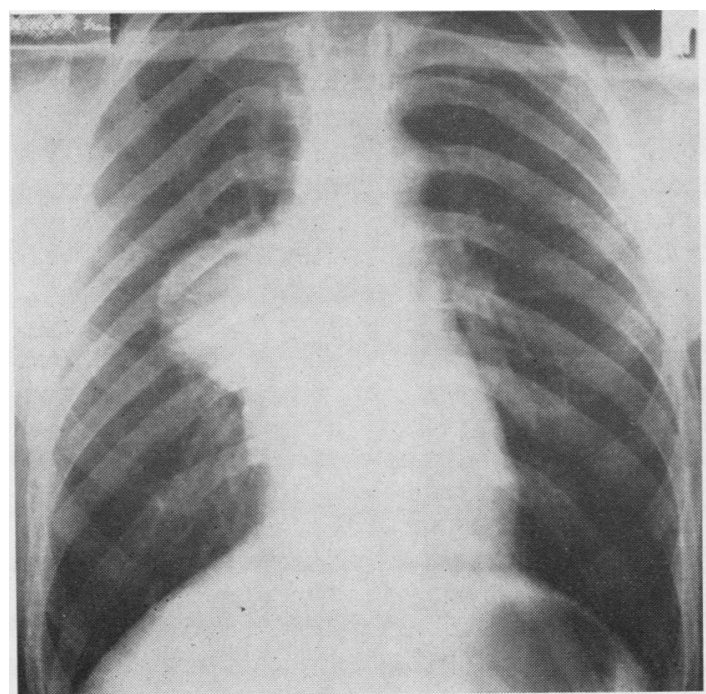

Fig. 3.-Postero-anterior view showing a mediastinal teratoma containing opacities of calcific density which were teeth.

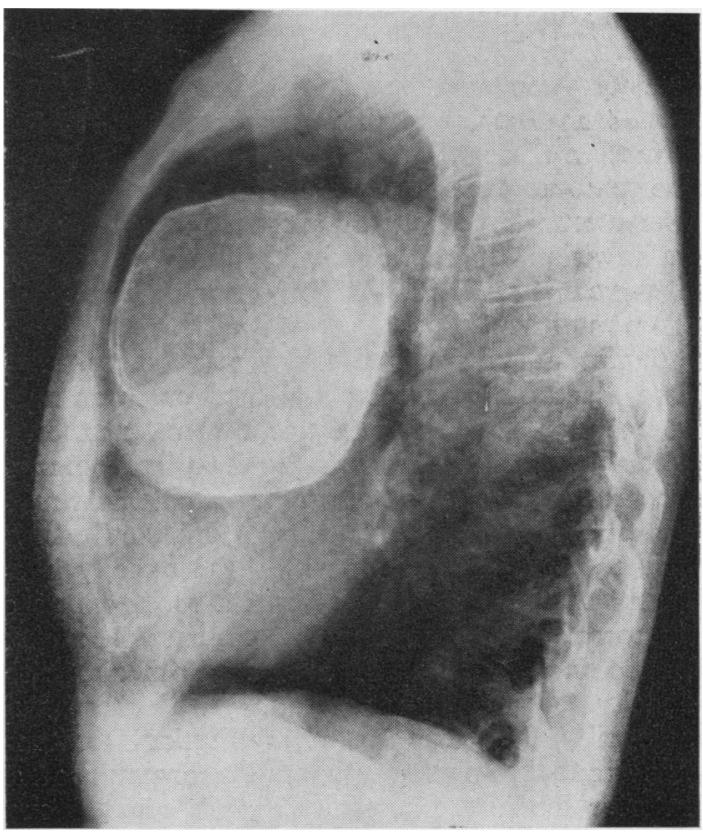

Fig. 2.-Left lateral view, showing the teratoma lying anteriorly.

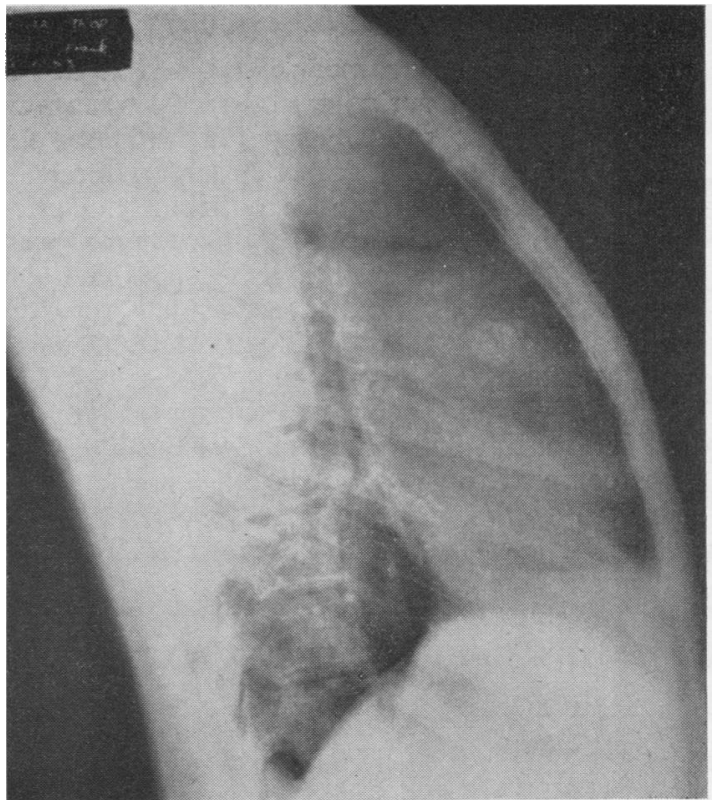

FIG. 4.-The teeth are more clearly seen in the lateral view. 
graphic opacity was outlined by a rind of calcium (Figs. 1 and 2). In two others, opacities of calcific density were seen within the lesion, were interpreted as teeth, and this interpretation was confirmed in the one resected (Figs. 3 and 4). Where the tumours were associated with visible deformity of the anterior chest wall, destruction by pressure or invasion of the overlying bone was neither observed radiographically nor found at thoracotomy.

Preliminary investigations were not helpful. A firm diagnosis of mediastinal teratoma was made in the two patients with trichoptysis, neither of whom showed either of the two radiographic features suggestive of teratoma, a calcific rim or teeth. In the six patients with the calcific rind, and in the one with calcium within the opacity interpreted as teeth, a firm pre-operative diagnosis of teratoma was also made. One patient with a chest wall deformity also had a calcific rim about the opacity. In the other four with chest wall deformity the diagnosis of mediastinal teratoma was made pre-operatively on the grounds of an anterior mediastinal opacity associated with such a deformity. In 12 of the 19 patients submitted to thoracotomy, therefore, a firm pre-operative diagnosis was made, and subsequently established histologically. In the remaining seven the diagnosis of anterior mediastinal teratoma was certainly included during pre-operative discussion, but there was no good reason for favouring this diagnosis over any of the other anterior mediastinal lesions, thymic tumours particularly. During the period in which the 19 teratomata were resected, 18 patients were submitted to thoracotomy for anterior mediastinal opacities histologically shown to be thymic lesions, seven malignant and 11 benign tumours (Figs. 5 and 6), three patients were submitted to thoracotomy for anterior mediastinal lesions shown histologically to be ectopic thyroids, and six other patients were submitted to thoracotomy for anterior mediastinal lesions two of which were reticulum-cell sarcomata, two lymphosarcomata (Figs. 7 and 8), and two Hodgkin's disease. All of these 27 patients had symptomless anterior mediastinal lesions found on routine radiography, and in each instance the diagnosis of teratoma had been included in the pre-operative discussions.

In three patients (Cases 3, 10, and 14) the anterior mediastinal opacity was visible on both sides of the mediastinum, but in each instance more of the opacity presented to one side, and there was never doubt regarding the appropriate side on which to make the thoracotomy. The tumours were all resected through a standard lateral thoracotomy, usually without rib resection. Eleven of the tumours were shelled out of the anterior mediastinum with a little blunt dissection, after the incision of the parietal pleural reflection over the tumour, and without the need for ligation of a pedicle. In four cases a pedicle from the superior mediastinum was ligated, was shown to contain a vessel of considerable calibre, $2-3 \mathrm{~mm}$., and in three of these a part of the pedicle was examined histologically and the contents were found to include normal thymic tissue. In two cases a branch of the pericardiaco-phrenic artery supplied the tumour and was ligated. The phrenic nerve was never involved, although it was usual to strip it off the tumour with the pleural reflection. In two instances the pericardium was partially resected because the tumour was densely adherent to it. In the two patients with trichoptysis, pulmonary communication with the tumour was established, in one with both lungs, and in the other with one lung. In the former the opposite pleural space was widely opened at thoracotomy and residual air leak from both lungs embarrassed early convalescence. In the latter, part of the anterior segment of the left upper lobe was amputated with the tumour and was shown to contain an acute inflammatory abscess cavity.

In seven patients with large tumours there was, after resection of the tumour, a residual dent in the inflated lung on the side of thoracotomy. This dent corresponded precisely with the site and shape of the resected tumour and the pulmonary deformity is regarded as evidence of a large teratoma having been present for a considerable time.

One of the tumours resected was very large (Case 10) and its pleural surface was ulcerated. This tumour was shown histologically to be malignant with predominantly adenocarcinomatous changes. The patient died three months later from diffuse metastases. The remaining 18 patients submitted to resection are well 11 years to 15 months after thoracotomy.

The histological features of the 18 benign teratomata are similar. Where calcium was seen radiographically its presence was confirmed histologically. The bony hardness of the tumours with a calcific rind was commented upon in many of the operation notes. In a large number of the tumours bronchial tissue was a prominent feature. Skin and adnexae, central nervous system tissues with choroid plexus, alimentary epithelium, cartilage, plain and striated muscle, pancreas, thymus, various unidentified glandular structures, and thyroid were all found. Hair when it occurred was grey, white, or black. There were cystic 

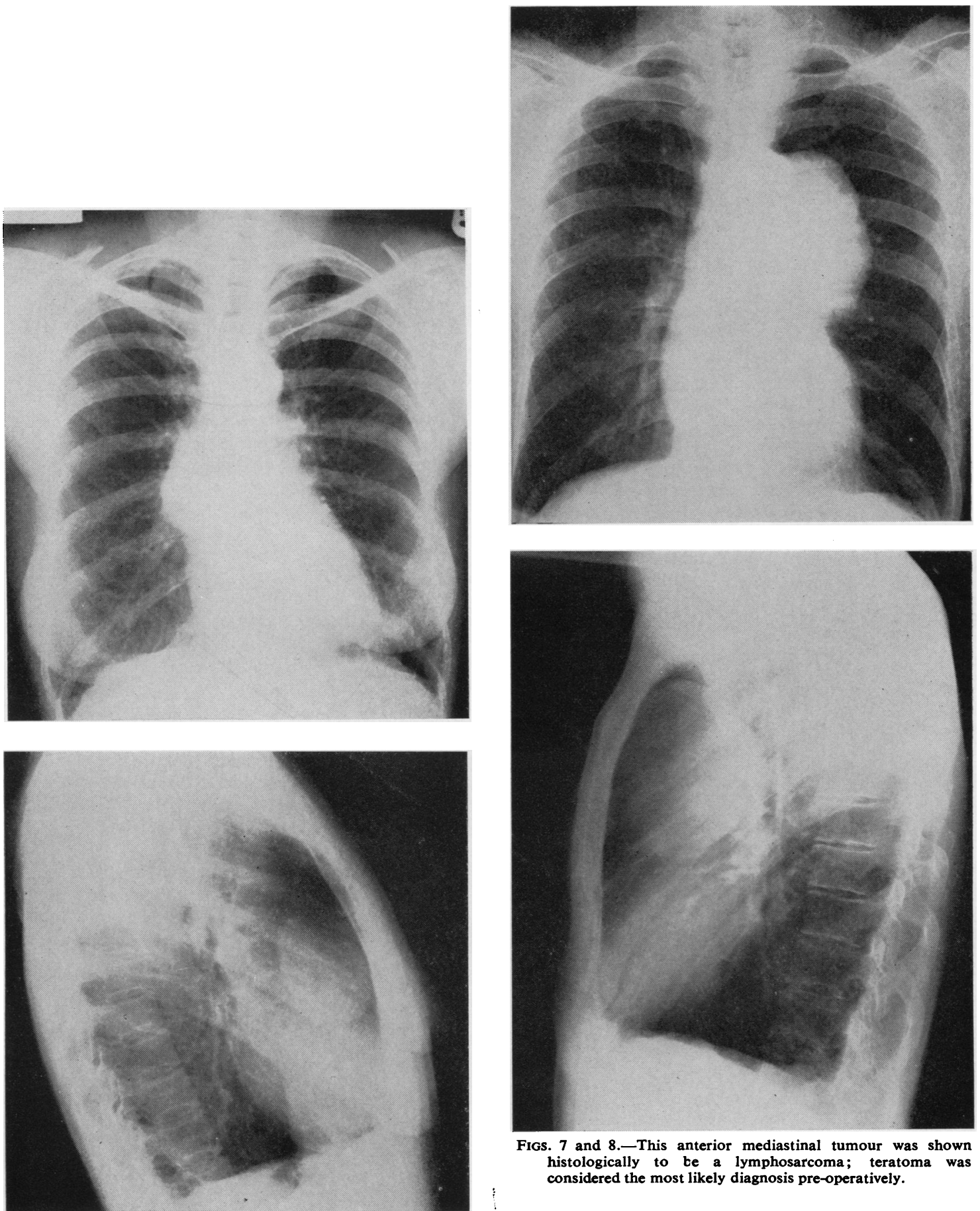

FIGs. 7 and 8.-This anterior mediastinal tumour was shown histologically to be a lymphosarcoma; teratoma was considered the most likely diagnosis pre-operatively.

FIGs. 5 and 6.-This anterior mediastinal tumour was shown histologically to be a benign thymic cyst; teratoma was included in the pre-operative differential diagnosis. 
spaces in all the tumours and in only two was , there a single large cyst. Both these contained "hair, one contained teeth, and in both the walls were relatively thin and composed of fibrous tissue with only occasional islets of muscle, cartilage, and sebaceous glands.

The Regional Thoracic Unit in Edinburgh serves a population of about $1 \frac{1}{2}$ million. During an average year one or two mediastinal teratomata present for the first time. The year 1958 was one of intense activity on the part of mass radiographic units in the South-East Region of Scotland and during this year five new teratomata were found.

It is customary to resect anterior mediastinal lumps whether or not the patient has symptoms. in some patients resection is undertaken because of uncertainty of the nature of the opacity, and in those where there is a reasonable basis for the diagnosis of teratoma resection is undertaken because of the complications which may develop, namely, infection, with or without bronchial communication, reduction of space within the chest and pressure effects, rupture into the pleura, the pericardium, or a bronchus, haemorrhage, and malignant change.

Distinction between a calcified aortic aneurysm and a teratoma with a calcific periphery is some- times possible on plain films in two views because the calcific shadow may so change its relationship to the aorta that origin from the aorta is unlikely. Where there is doubt it may be necessary to make angiograms. In only one of the 19 patients submitted to thoracotomy for a lesion shown to be a teratoma was the diagnosis of aneurysm seriously considered and excluded by angiography.

\section{SUMMARY}

Mediastinal teratomata are true tumours. Their histogenesis is outlined. Twenty-one new cases are described, 19 of which were proven histologically. The presence of teeth within or a calcific rind about an anterior mediastinal opacity suggests the diagnosis of teratoma; deformity of the anterior chest wall in relation to an anterior mediastinal opacity is similarly suggestive. Two patients had coughed up hair: one has died from metastasis from a malignant teratoma; 20 are well.

\section{REFERENCES}

Morrison, I. M. (1958). Thorax, 13, 294.

Schlumberger, H. G. (1951). Atlas of Tumor Pathology, Section V, Fascicle 18. Armed Forces Institute of Pathology, Washington. Willis, R. A. (1953). Pathology of Tumours, 2nd ed. Butterworths, London. 